\title{
Challenges in Managing Pregnancy with Complete Heart Block and Its Outcome in a Tertiary Center in Nepal
}

\author{
Pooja Paudyal $^{1} \odot$, Suniti J Rawal ${ }^{2}$, Prabhat Khakural $^{3} \odot$
}

\begin{abstract}
Aim:Various congenital and acquired causes can lead to abnormal conduction of electrical impulses from atria to ventricles, resulting in complete atrioventricular dissociation. Such complete heart blocks (CHBs) are uncommon in pregnant women. However, when diagnosed in pregnancy, the management remains challenging. Here, we share our experience of managing patients with CHB diagnosed in pregnancy and their outcome. Materials and methods: A retrospective review of women diagnosed with $\mathrm{CHB}$ during pregnancy and managed in the Department of Obstetrics and Gynecology, Tribhuvan University Teaching Hospital, and in Manmohan Cardiothoracic and Vascular Center, from April 2016 to March 2020, was done. Data studied from case records included history, clinical examination, 12-lead electrocardiogram, echocardiogram, 24-hour Holter study, exercise tolerance, response to chronotropic drugs, and antenatal follow-up. Mode of delivery, need for pacing, and maternal and fetal outcomes were evaluated.

Results: Six women had CHB diagnosed between 22 and 40 weeks' gestation of index pregnancy. The mean age of the patients was $25.8 \pm 5.1$ years. The heart rate at diagnosis was between 40 and $48 \mathrm{bpm}$. None of the patients had any symptoms or abnormal echocardiography. Fifty percent had vaginal delivery. All of the patients had an uneventful peripartum period and none had any emergency indication for pacing. The mean birth weight of the babies was $2485.7 \pm 389 \mathrm{~g}$ and two of them had intrauterine growth retardation. None of the babies had any conduction or cardiac structural abnormalities.

Conclusion: Close monitoring, periodic follow-up, and vigilant management by a multidisciplinary team of obstetricians, cardiologists, anesthetists, and pediatricians in a center with the facility of insertion of a cardiac pacemaker can ensure a good maternal and fetal outcome in pregnant women with $\mathrm{CHB}$.

Clinical significance: Asymptomatic CHB in pregnancy can be managed conservatively without pacing.

Keywords: Complete heart block, Pacemaker, Pregnancy.

Journal of South Asian Federation of Obstetrics and Gynaecology (2020): 10.5005/jp-journals-10006-1834
\end{abstract}

\section{INTRODUCTION}

Complete heart block (CHB) or complete atrioventricular block (CAVB) is a disorder of cardiac conduction. There is a random relationship between atrial and ventricular activation where atrial impulses fail to get conducted to ventricles. ${ }^{1,2}$ CAVB can be either congenital or acquired secondary to cardiac surgery, infective disorders, and autoimmune diseases. ${ }^{1}$

CAVB could be life-threatening with risk of Stokes-Adams (SA) attacks and sudden death. ${ }^{3}$ It is more prevalent in elderly male patients with ischemic or sclerodegenerative heart disease and is rarely encountered in pregnant women. ${ }^{1,4}$ CAVB poses a significant challenge in the management during pregnancy and labor due to the normal physiologic alterations of pregnancy which may lead to cardiovascular decompensation, more so when first diagnosed during pregnancy. As pregnancy and $\mathrm{CHB}$ is a rare association and reported in the literature as small series or isolated case reports, there are no established guidelines regarding optimal management. ${ }^{1-6}$ We therefore report our experience and outcome of six patients with $\mathrm{CHB}$ detected during pregnancy and discuss the challenges in their management.

\section{Materials and Methods}

This was a retrospective review of cases managed over 5 years from April 2016 to March 2020 in the Department of Obstetrics and Gynecology, Tribhuvan University Teaching Hospital, and

\footnotetext{
1,2 Department of Obstetrics and Gynaecology, Tribhuvan University Teaching Hospital, Kathmandu, Nepal

${ }^{3}$ Department of CTVS, Manmohan Cardiothoracic Vascular and Transplant Centre, Kathmandu, Nepal
}

Corresponding Author: Pooja Paudyal, Tribhuvan University Teaching Hospital, Department of Obstetrics and Gynaecology, Kathmandu, Nepal, Phone: +977 9841526853, e-mail: paudyalpooja@yahoo.com

How to cite this article: Paudyal P, Rawal SJ, Khakural P. Challenges in Managing Pregnancy with Complete Heart Block and Its Outcome in a Tertiary Center in Nepal. J South Asian Feder Obst Gynae 2020;12(6):359-362.

Source of support: Nil

Conflict of interest: None

in Manmohan Cardiothoracic and Vascular Center. All pregnant women who were first diagnosed to have $\mathrm{CHB}$ during pregnancy were studied. All cases had been subjected to detailed evaluation including history and review of historical data from case records, clinical examination, 12-lead electrocardiogram (ECG), and echocardiography (ECHO). Patients were monitored with a 24-hour ECG Holter and exercise tolerance was evaluated and their response to chronotropic drugs, such as atropine was tested. To rule out connective tissue disorder as a cause for $\mathrm{CHB}$, a collagen profile was performed in all patients. Patients were followed at regular intervals throughout the pregnancy by the obstetric team and 
periodic cardiac evaluation was performed by the cardiology team. Serial ultrasonography was performed to monitor the growth of the fetus and a modified biophysical profile was carried out to monitor growth-retarded fetuses. Patients were allowed to go into spontaneous labor or plan for an elective termination according to their obstetric indications. A cesarean section (CS) was performed for obstetric indications only. Patients were closely monitored during labor with continuous cardiac monitoring for heart rate, periodic blood pressure, and clinical cardiac examination for heart failure. Epidural anesthesia was electively used to minimize the hemodynamic changes arising from pain or bearing down. Patients were managed conservatively without pacing during labor and delivery or CS, albeit cardiologists with facilities for temporary pacing were on standby. The cases were analyzed with respect to clinical profile, maternal and fetal morbidity, and treatment given during the pregnancy and delivery.This study was ethically cleared from the institutional review committee.

\section{Results}

During the 5 years, six patients with $\mathrm{CHB}$ delivered in our institute. All the six patients were diagnosed to have $\mathrm{CHB}$ during the index pregnancy. Four patients were nulliparous while two were multipara. One of the women had twin gestation and the rest were singleton pregnancies. Five were referred whereas one was our booked case. The mean age of the patients was $25.8 \pm 5.1$ years. They were diagnosed to have $\mathrm{CHB}$ earliest at 22 weeks of gestation and latest at 40 weeks. Case 2 was diagnosed on the operating table after connecting to the ECG monitor; case 3 was diagnosed during preanesthetic checkup prior to the lower segment cesarean section (LSCS) while the other four cases were diagnosed by the obstetrician during routine antenatal visit on palpating the pulse. The heart rate at diagnosis was between 40 and 48 bpm; none of the patients had any symptoms of CHB and all had essentially normal echocardiography findings with no underlying structural heart disease (Tables 1 and 2).
The collagen profile (rheumatoid factor, antinuclear antibody antineutrophilic cytoplasmic antibody, and anticardiolipin antibodies) was negative in all patients.The thyroid function test in all women was normal. All the women were evaluated by the cardiologist for the need for pacing. In view of the patients being asymptomatic and showing good chronotropic competence, they were planned to deliver without pacing.

All of the women delivered at term. Fifty percent had vaginal delivery, among which two were spontaneous in onset and one was induced due to reduced fetal movements at 38 weeks while the rest $50 \%$ had a CS. A CS was done for obstetric indications-case 1: intrauterine growth restriction (IUGR) with unstable lie; case 2: premature rupture of membranes with twin pregnancy; and case 3: severe IUGR with postdated pregnancy. Epidural analgesia was given for all undergoing vaginal delivery. CS was done with combined spinal-epidural anesthesia in case 1 and under general anesthesia in cases 2 and 3 . All of the patients had an uneventful intrapartum period, the blood pressures remained normal and the heart rates did not alter significantly during labor, and none had any emergency indication for pacing.

The mean birth weight of the babies was $2485.7 \pm 389 \mathrm{~g}$. Two cases had IUGR babies with $2.2 \mathrm{~kg}$ and $1.8 \mathrm{~kg}$ while the rest of the five babies were appropriate for gestational age. The $1.8 \mathrm{~kg}$ baby was admitted to the neonatal intensive care unit with a diagnosis of meconium aspiration syndrome and small for gestational age with early-onset neonatal sepsis for 7 days. The rest of the babies had good APGAR score and were healthy (Table 2). None of the babies had any rhythm or structural abnormalities of the heart on evaluation following delivery.

\section{Discussion}

$\mathrm{CHB}$ encountered during pregnancy is a rare occurrence; it was seen in 2.5 per 10,000 deliveries $(6 / 23,385)$ in our center. Suri et al. describe their findings of four women over a span of 4 years; Mandal et al. studied 21 patients in 9 years with an incidence of

Table 1: Clinical profile of the patients

\begin{tabular}{llllllll}
\hline Sl. No. & Age & Parity & $\begin{array}{l}\text { Time of diagnosis (gestational } \\
\text { age in weeks) }\end{array}$ & $\begin{array}{l}\text { Heart rate at diagnosis } \\
(\mathrm{bpm})\end{array}$ & Cardiac symptoms & Echo finding & Booked/referred \\
\hline 1 & 24 & Nulli & 32 & 46 & None & Mild MR, TR & Referred \\
2 & 23 & Nulli & 39 & 42 & None & Normal & Booked \\
3 & 24 & Nulli & 40 & 44 & None & Normal & Referred \\
4 & 30 & Nulli & 29 & 48 & None & Normal & Referred \\
5 & 20 & Multi & 22 & 40 & None & Normal & Referred \\
6 & 34 & Multi & 35 & 46 & None & Normal & Referred \\
\hline
\end{tabular}

Table 2: Labor and delivery events with obstetrical outcomes

\begin{tabular}{|c|c|c|c|c|c|c|c|}
\hline SI. No. & $\begin{array}{l}\text { Type of } \\
\text { pregnancy }\end{array}$ & $\begin{array}{l}\text { Gestational age at delivery } \\
\text { (weeks) }\end{array}$ & Labor onset & Mode of delivery & Birth weight $(\mathrm{kg})$ & Analgesia/anesthesia & $\begin{array}{l}\text { Complication } \\
\text { (maternal/fetal) }\end{array}$ \\
\hline 1 & Singleton & 37 & Elective CS & LSCS & 2.2 & Epidural & IUGR \\
\hline 2 & Twin & 39 & Spontaneous & LSCS & 2.5, each & General & - \\
\hline 3 & Singleton & 41 & Elective CS & LSCS & 1.8 & General & IUGR \\
\hline 4 & Singleton & 38 & Spontaneous & Vaginal delivery & 2.7 & Epidural & - \\
\hline 5 & Singleton & 39 & Spontaneous & Vaginal delivery & 2.7 & Epidural & - \\
\hline 6 & Singleton & 38 & Induced & Vaginal delivery & 3 & Epidural & - \\
\hline
\end{tabular}


2.4/1,000 deliveries; and Thaman et al. reported 18 cases over 11 years. ${ }^{1,2,6} \mathrm{CHB}$ in pregnancy being so rare, there are only single case reports or studies with a small number of cases and there is no randomized controlled trial. Hence, there is no universal protocol for the management and when $\mathrm{CHB}$ is encountered in a pregnant woman it is a challenge not only for the obstetrician but also for the cardiologist and the anesthetist as well. The need for pacing, mode of delivery, and anesthetic methods are all debatable.

\section{Age, Parity, and Type of Gestation}

The mean age was 25.8 years similar to findings of 25.5 years by Suri et al. ${ }^{1}$ In our study, four women were nulliparous and two were multiparous whose $\mathrm{CHB}$ was undetected in their previous deliveries. Keepanasseril et al. describe seven pregnancies in a woman with $\mathrm{CHB}$ without any major complications. ${ }^{7}$ One of our patients had twin gestation. Multiple gestations in women with CAVB have been reported in two case reports with authors suggesting that the cardiac workload in multiple pregnancies is greater than that of in a singleton pregnancy and the increased maternal heart rate and contractility in multiple pregnancies leads to a greater reduction in the cardiovascular reserve., 8 But our patient had no features of cardiac decompensation throughout the pregnancy.

\section{Diagnosis of CHB}

All the six women in our study were diagnosed to have $\mathrm{CHB}$ during their present pregnancy antenatally. Similarly, in the study by Suri et al., all four women were diagnosed during pregnancy while other studies have described women with previously diagnosed $\mathrm{CHB}$ along with the newly diagnosed ones during antenatal, intranatal or postnatal period. ${ }^{1,2,5,6}$

The earliest diagnosis in our study was made at 22 weeks, following which the patient was referred to us. One case was diagnosed prior to emergency LSCS and one during preanesthetic checkup for an elective LSCS, after which she was referred to our center. Baghel et al. described a woman at 42 weeks 3 days who was diagnosed during labor. ${ }^{10}$ Here we cannot undermine the importance of palpating the pregnant woman's pulse during antenatal checkup as most women are diagnosed simply by a clinical finding of a low pulse rate. If diagnosed during antenatal care, the labor and delivery could have been planned and managed well in advance and would not have created a last-minute havoc due to diagnosis during labor.

\section{Symptoms and Echocardiography Findings}

All six women were asymptomatic and diagnosed only with an incidental finding of a low pulse rate. Thirty percent of the cases of congenital $\mathrm{CHB}$ remain undiagnosed as they are asymptomatic until adulthood and may therefore present during pregnancy. ${ }^{11}$ In most of the studies, the majority of women were asymptomatic during pregnancy ${ }^{1,4,5,6}$ except for Mandal et al. who reported 21 pregnant women with $\mathrm{CHB}$ and found all of them to be symptomatic with syncope and palpitation seen in 29 and $38 \%$ women, respectively. ${ }^{2}$

None of our patients had underlying structural heart disease on echocardiography; of the four patients reported by Suri et al., one case had corrected transposition of great arteries; Mandal et al. detected a single ventricle in one and corrected transposition of great arteries in two cases., ${ }^{1,2}$ Hidaka et al. reported a history of SA attack in one of the six patients and none had any structural cardiac diseases. ${ }^{4}$ Thus, it is important to evaluate all women with low pulse rate even though they are asymptomatic and also to rule out any underlying heart disease.

\section{Delivery}

The choice of mode of delivery was based on the obstetric indications only. Three delivered vaginally while three had LSCS for obstetric indications. The presence of $\mathrm{CHB}$ does not determine the mode of delivery as vaginal delivery has been found to be successful.

Suri et al. reported that two of their four patients had a CS for fetal indication, whereas the other two had an uneventful vaginal delivery. ${ }^{1}$ Mandal et al. reported $86 \%$ vaginal deliveries and Hidaka et al. describe successful vaginal delivery in all six women. ${ }^{2,4}$ Augmentation of labor to shorten the active phase of labor and elective instrumental delivery to cut short the second stage has been recommended as these women are prone to develop syncopal attacks and convulsions due to slowing of the heart rate associated with the Valsalva maneuver during forceful uterine contractions. ${ }^{2-6}$ Though the second stage was not cut short with instrumental delivery in our patients, they withstood the stress of labor without any adverse events similar to the findings of Suri et al. ${ }^{1}$

Continuous cardiac monitoring was done during the intrapartum period and the heart rate and blood pressure remained stable. For all women, the maternal heart rate did not significantly change before, during or after labor and delivery.

The three women who had delivered vaginally were administered epidural analgesia in this study. In normal labor without epidural anesthesia, cardiac output during contractions increases progressively as labor advances and increases by a mean value of $34 \%$ at full cervical dilatation. ${ }^{8}$ Epidural anesthesia reduces these changes in addition to reducing labor pain and the studies have recommended that epidural analgesia is helpful in parturient with CAVB. ${ }^{1,2,4,5}$

\section{Obstetric Complications}

The pregnancy and delivery were without any major maternal or fetal complications though IUGR was present in two of our cases. Hidaka et al. report IUGR in 2 of 20 pregnancies with CHB without pacemaker but was not statistically significant. ${ }^{5}$ Theoretically, reduced uteroplacental supply due to low heart rate and low cardiac output is the possible cause of fetal growth restriction. Preterm labor has been seen in some studies. ${ }^{1,2}$ There was no obvious difference in the frequency of obstetric complications between cases with pacemaker and cases without pacemaker; therefore, pacemaker insertion to avoid obstetric complications was not advised. ${ }^{5}$

\section{Pacing}

The requirement for pacing in symptomatic patients has been clearly demonstrated. However, there is still room for discussion in determining who to pace and when to pace if the patient is asymptomatic. ${ }^{5}$ The role of placement of a pacemaker in asymptomatic women is debatable.

A study by Michaelsson et al. found that there was a high incidence of SA attacks with increased mortality in asymptomatic adults and also an increase in the incidence of mitral regurgitation along with the development of cardiomyopathy in the long term, hence a prophylactic pacemaker insertion was recommended. ${ }^{3} \mathrm{~A}$ temporary pacemaker (TPM) in a laboring woman could prevent extreme bradycardia by compensating any possible hemodynamic changes that occur during labor, particularly during the second stage following anesthesia and the immediate postpartum period. ${ }^{10}$ Mandal et al. recommend delivery under pacemaker coverage 
to mitigate the risks of sudden bradyarrhythmia. They reported delivery with pacemaker (either temporary or permanent) in 16 of 21 cases and 5 required pacing postpartum. Indications of pacing included maternal bradycardia nonresponsive to medication and in patients with left ventricular dysfunction and presence of history of syncope in the preconception, antenatal, and postnatal period. ${ }^{2}$

Other experts on the contrary recommend pacemaker implantation should be delayed as long as possible in young asymptomatic patients, although the risk of sudden death is unpredictable because of the risk of infection and the requirement for replacement of the pacemaker. But if pregnant women with CAVB become symptomatic at or near term, they recommend temporary pacing. ${ }^{5}$ Suri et al. had an experience of four pregnancies with $\mathrm{CHB}$ and observed two patients requiring temporary pacing and the rest two who delivered without the support of a pacemaker. ${ }^{1}$

Thaman et al. reported that of the four patients with CHB first diagnosed during pregnancy two required permanent pacemaker as they were symptomatic but the other two were followed up closely and did not require pacing and did not experience significant bradyarrhythmia during labor or at the time of delivery. ${ }^{6}$ Hidaka et al. concluded that women with AV block and without permanent pacing do not routinely require temporary pacing for labor and birth. They had precautionary elective insertion of a temporary lead to be used only if the patient becomes symptomatic but none of the patients required pacing during labor and delivery. ${ }^{4}$

Because of all these contrasting findings, whether pacing in $\mathrm{CHB}$ with pregnancy is the necessary step of management or not is a debatable issue. In coordination with the team of cardiologists, our patients were managed during labor and delivery without pacing as all our patients were asymptomatic and exhibited exercise tolerance and were responsive to drugs like atropine. But the availability of the cardiologist and TPM was ensured, if the need arose.

\section{Conclusion}

There are lots of issues and challenges in managing CAVB in pregnancy which is encountered few and far between in clinical practice. They need multidisciplinary management with the involvement of obstetricians, cardiologists, anesthetists, and pediatricians. Close monitoring and follow-up and preparedness is the key to good management along with periodic evaluation for the need for pacing by a cardiologist. These patients usually have a good pregnancy outcome and tolerate labor and delivery well. The mode of delivery is as per the obstetric indications. Though asymptomatic patients with exercise tolerance can be managed without a pacemaker in pregnancy and delivery, there should be an available arrangement and the facility of insertion of TPM if the need arises.

\section{Limitations}

The retrospective nature of the study and the small number of cases are the limitations of this study and a larger study is warranted for further exploration of this unique condition in pregnancy.

\section{Clinical Significance}

While delivery under cover of a pacemaker is a safe option in pregnant women with $\mathrm{CHB}$, conservative management without pacing in asymptomatic women may be a practical alternative.

\section{OrCID}

Pooja Paudyal @ic https://orcid.org/0000-0002-7522-0759

Suniti J Rawal @ https://orcid.org/0000-0003-0244-6922

Prabhat Khakural @ i https://orcid.org/0000-0003-2057-4792

\section{References}

1. Suri V, Keepanasseril A, Aggarwal N, et al. Maternal complete heart block in pregnancy: analysis of four cases and review of management. J Obstet Gynaecol Res 2009;35(3):434-437. DOI: 10.1111/j.14470756.2008.00961.x.

2. Mandal S, Mandal D, Sarkar A, et al. Complete heart block and pregnancy outcome: an analysis from Eastern India. SOJ Gynaecol Obstet Womens Health 2015;1(1):5. DOI: 10.15226/2381-2915/1/1/00104.

3. Michaëlsson $M$, Jonzon A, Riesenfeld T. Isolated congenital complete atrioventricular block in adult life. A prospective study. Circulation 1995;92(3):442-449. DOI: 10.1161/01.cir.92.3.442.

4. Hidaka N, Chiba Y, Kurita T, et al. Is intrapartum temporary pacing required for women with complete atrioventricular block? An analysis of seven cases. BJOG 2006;113(5):605-607. DOI: 10.1111/j.14710528.2006.00888.x.

5. Hidaka N, Chiba Y, Fukushima K, et al. Pregnant women with complete atrioventricular block: perinatal risks and review of management. Pacing Clin Electrophysiol 2011;34(9):1161-1176. DOI: 10.1111/j.15408159.2011.03177.x.

6. Thaman R, Curtis S, Faganello G, et al. Cardiac outcome of pregnancy in women with a pacemaker and women with untreated atrioventricular conduction block. Europace 2011;13(6):859-863. DOI: 10.1093/europace/eur018.

7. Keepanasseril A, Maurya DK, Suriya Y, et al. Complete atrioventricular block in pregnancy: report of seven pregnancies in a patient without pacemaker. BMJ Case Rep. 2015;2015. DOI: 10.1136/bcr-2014-208618.

8. Ramsewak S, Persad P, Perkins S, et al. Twin pregnancy in a patient with complete heart block. A case report. Clin Exp Obstet Gynecol 1992;19(3):166-167. PMID: 1451281

9. Avasthi K, Gupta S, Avasthi G. An unusual case of complete heart block with triplet pregnancy. Indian Heart J 2003;55:641-642. PMID: 14989517.

10. Baghel K, Mohsin Z, Singh S, et al. Pregnancy with complete heart block. J Obstet Gynaecol India 2016;66(2):623-625. DOI: 10.1007/ s13224-016-0905-z.

11. Dalvi BV, Chandhuri A, Kulkarni HL, et al. Therapeutic guidelines for congenital complete heart block. Obstet Gynecol 1992;79:802-804. PMID: 1565369 